\title{
LA IMPORTANCIA DEL EMPRENDIMIENTO SOCIAL FRENTE AL PROCESO DE GLOBALIZACIÓN DE LA ECONOMÍA Y LOS MERCADOS
}

\author{
THE IMPORTANCE OF SOCIAL ENTREPRENEURSHIP TO THE PROCESS \\ OF GLOBALIZATION OF THE ECONOMY AND MARKETS
}

Francisca Bouby Tolentino*
f_bouby@yahoo.com

[RECEPCIÓN: MARZO DE 2015 / CONFORMIDAD: ABRIL DE 2015]

\begin{abstract}
RESUMEN
El objetivo del presente trabajo es contribuir al debate que permita profundizar en el análisis de por qué la separación entre ética y ciencia económica ha conllevado a olvidar la dimensión altruista del ser humano y a considerar solamente su dimensión egoísta, la misma que ha perfilado un sistema económico global con problemas de desigualdad, pobreza, exclusión social y medio ambientales. El análisis crítico de los diversos documentos revisados en relación a este debate y los indicadores resultantes, conducen a plantear la importancia del emprendimiento social, vinculado a la consecución de un sistema económico más justo donde ética y economía vayan unidas. El emprendimiento social está surgiendo como una respuesta a los problemas que está generando el modelo de capitalismo global a la población y para cubrir el vacío de las débiles políticas sociales de los gobiernos enfocados en políticas de desregulación y liberalización Económica. El emprendimiento social constituye un modelo empresarial complementario al emprendimiento económico o de mercado y al sistema empresarial corporativo existente, al actuar en áreas donde no suele actuar el emprendimiento de mercado, por lo cual puede contribuir a mejorar el sistema económico global perfilando un sistema más justo y eficiente.
\end{abstract}

Palabras clave: Globalización, emprendimiento económico, emprendimiento social, ética.

\begin{abstract}
The objective of the present paper is to contribute to the debate that will allow us to deepen the analysis of why the separation between ethics and economic science has led us to forget the altruistic dimension of human being and consider only its selfish dimension, the same that has shaped a global economic system with problems of inequality, poverty, social exclusion and environmental disarray. The critical analysis of the various reviewed documents in relation to this debate and
\end{abstract}

\footnotetext{
* Doctora en Economía (UNAM, México, D.F.), magíster en Economía con mención en Política Económica (UNMSM), docente principal a nivel pre y post grado e investigadora en el Instituto de Investigación de la Facultad de Ciencias Administrativas (FCA) (UNMSM). Ex directora de la Escuela Académico Profesional de Administración de Turismo de la FCA, (UNMSM). Ex Vicerrectora Académica de la Universidad SISE.
} 
the resulting indicators, lead us to raise the importance of social entrepreneurship, linked to the achievement of a more fair economic system where ethics and economy are inextricably linked. Social entrepreneurship is emerging as a response to the problems that the model of global capitalism is generating to the population and to fill the vacuum of weak social policies of governments focusing on policies of deregulation and economic liberalization. Social entrepreneurship constitutes a supplementary business model to the economic or market entrepreneurship and to the existing corporate enterpreneural system, by way of acting in áreas where market enterpreneurship usually doesn't act. Therefore, it can contribute in the improvement of the global economic system, giving shape alltogether to a more fair and efficient system.

Keywords: Globalization, economic entrepreneurship, social entrepreneurship, ethics.

\section{INTRODUCCIÓN}

\section{Planteamiento del problema}

La economía global se ha ido transformando durante las últimas décadas, configurándose actualmente un nuevo modelo, basado fundamentalmente en la competencia y la nueva tecnología. Este modelo no ha logrado resolver los problemas generados en la economía global, por el contrario los ha agudizado.

Y es que el sistema capitalista enfatiza la dimensión egoísta del comportamiento humano y prescinde de otras dimensiones como la altruista o la cooperativa, lo cual conduce a configurar un sistema económico global caracterizado por la competencia y el afán de lucro, con el objetivo de maximizar beneficio coherente con la lógica de una conducta egoísta.

Este sistema de capitalismo global caracterizado por un crecimiento cíclico de auge y crisis, viene generando grandes problemas de orden económico y social. Los cuales han dado como resultado una elevada desigualdad social, crecimiento de la pobreza, desempleo y exclusión social, fundamentalmente en los países en desarrollo,

Esta situación está contribuyendo a la cada vez mayor polarización a nivel de la economía mundial por el deterioro de las condiciones de vida de inmensas capas de la población a nivel global.

La importancia del emprendimiento social, está vinculada a la consecución de un sistema económico más justo en el cual la ética y la economía van unidas. El emprendimiento social está surgiendo como una respuesta a los problemas que está generando el modelo de capitalismo global a la población, fundamentalmente en términos de desigualdad, pobreza y problemas medio ambientales; y para cubrir el vacío de las débiles políticas sociales de los gobiernos enfocados en políticas de desregulación y liberalización Económica.

\section{Relación del estudio con otros similares}

Castellanos (2003), en su artículo Propuesta de formación en liderazgo y emprendimiento sostiene que las sociedades cada vez más cambiantes caracterizan al naciente siglo. En ellas, el fortalecimiento del emprendimiento es determinante como elemento transversal en la formación no sólo de profesionales monodisciplinares sino de personas aptas para incorporarse en las dinámicas del conocimiento y desarrollo socioeconómico de países con profundas necesidades de cambio.

Chaparro-Africano (2010), en su artículo "Emprendimientos y empresas agrarias y rurales más sustentables", argumenta que para la sustentabilidad de los territorios, argumenta que el emprendimiento y la empresa por sí mismos no son la solución ante la demanda de riqueza y progreso para paliar la pobreza, la inequidad y la insustentabilidad. De hecho la empresa ha agudizado estos indicadores sociales y ecológicos dadas sus características convencionales como las relaciones capitalistas de producción, centradas en la maximización y acumulación de capital, y su visión de industrialización que implica una masiva extracción de recursos y generación de desechos.

Garcíay Piña-Zambrano (2013), en su artículo Institucionalidad venezolana sobre emprendimiento social durante el periodo 1999-2010, destaca que, en ese período, se ha creado un entorno institucional para fomentar el emprendimiento social, constituido principalmente por normas jurídicas y políticas públicas orientadas a priorizar la integración de los aspectos económicos y sociales, con alta participación de organizaciones comunitarias y fuerte intervención estatal en las áreas financiera, técnica y de capacitación, con el propósito de alcanzar el desarrollo endógeno y humanista como modelo de diversificación de la economía, bajo parámetros de sustentabilidad. 
García (2014), en su artículo Bioética, emprendimiento e innovación en el contexto latinoamericano, argumenta que el emprendimiento y la innovación se han convertido en importantes instrumentos de desarrollo económico de manera que se buscó establecer en qué medida el emprendimiento y la innovación han considerado el componente bioético en su contenido, bajo un método cualitativo de corte documental.

Guzmán y Trujillo (2008), en su artículo "Emprendimiento Social - Revisión de Literatura", argumentan que el emprendimiento ha cobrado interés para las escuelas de negocios desde la década de los 80 , debido a la relación con la creación y dirección de empresas, entre otras razones. El emprendimiento es un campo vasto que involucra diferentes tópicos como financiación del emprendimiento, características del emprendedor, emprendimiento corporativo, empresas de familia, reconocimiento de oportunidades, aprendizaje y emprendimiento social, entre otros.

Santos (2013), en su artículo "Economía global y emprendimientos sociales", estudia el papel que pueden desempeñar los emprendimientos sociales en la consecución de un sistema económico mundial más justo.

Toca (2010), en su artículo "Consideraciones para la formación en emprendimiento: explorando nuevos ámbitos y posibilidades", propone una reflexión sobre la importancia del emprendimiento para el desarrollo eficiente y competitivo de las sociedades, tomando como punto de partida los diferentes conceptos que se han adoptado, a fin de resaltar su carácter transdisciplinar.

Wompner (2010), en su artículo El emprendimiento como factor de movilidad social, propone una reflexión sobre la importancia del emprendimiento para el desarrollo eficiente y competitivo de las sociedades, tomando como punto de partida los diferentes conceptos que se han adoptado, a fin de resaltar su carácter transdisciplinar.

\section{Justificación}

Estamos viviendo una situación contradictoria. Por un lado, hay un importante desarrollo de la ciencia y tecnología, y la globalización tecnológica permite beneficiar a ciertos sectores de la sociedad pero, al mismo tiempo, existen grandes brechas sociales y de exclusión que separan a una gran mayoría; por ello, es necesario plantear ideas renovadoras y contribuir a la generación de nuevos actores sociales, es decir, emprendedores sociales, comprometidos con el desarrollo de aquellos sectores de la población más vulnerables que no tienen acceso a servicios de calidad como salud, educación, entre otros.

\section{Objetivo del estudio}

El objetivo del presente trabajo es contribuir al debate que permita profundizar en el análisis de por qué la separación entre Ética y Ciencia Económica ha conllevado a olvidar la dimensión altruista del ser humano y a considerar solamente su dimensión egoísta, la misma que ha perfilado un sistema económico global con problemas de desigualdad, pobreza, exclusión social y medio ambientales. El emprendimiento social está surgiendo como una respuesta a los problemas que está generando el sistema, y para cubrir el vacío de las débiles políticas sociales de los gobiernos enfocados en políticas de desregulación y liberalización Económica. La importancia del emprendimiento social, está vinculada a la consecución de un sistema económico más justo donde ética y economía vayan unidas.

\section{Métodos}

El presente estudio se abordó bajo el enfoque cualitativo, como un estudio documental conformado por dos fases; una descriptiva y otra explicativa. La primera consistió en examinar la información cuantitativa y cualitativa existente sobre los efectos económico-sociales y medio ambientales del modelo económico que sustenta el sistema global vigente. La fase explicativa se generó a través de la revisión documental como técnica para profundizar en el debate orientado a construir explicaciones para analizar el papel del emprendimiento social, como como una respuesta a los problemas que está generando el modelo de capitalismo global a la población.

\section{RESULTADOS Y DISCUSIÓN}

\section{El proceso de globalización de la economía y de los mercados}

\section{Causas y efectos de las crisis del capitalismo global}

La economía global se ha ido transformando durante las últimas décadas, configurándose actualmente un nuevo modelo, basado fundamentalmente en la competencia y la nueva tecnología. 
Este modelo no ha logrado resolver los problemas generados en la economía global, por el contrario los ha agudizado.

En el trabajo de Santos se plantean dos nuevos debates que permiten profundizar en el diagnóstico de los problemas que aquejan a la nueva economía global y al modelo económico que la sostiene. El primer debate se ubica en el plano egoísmo-altruismo y el segundo en el plano beneficio financiero-atención a los intereses sociales (Santos 2013).

En el debate egoísmo-altruismo se busca explicar por qué la separación entre ética y ciencia económica ha conllevado a olvidar la dimensión altruista del ser humano y a considerar solamente su dimensión egoísta, la misma que ha ido perfilando el sistema económico global. Por otro lado, en el debate beneficio financiero-atención a intereses sociales, se trata de explicar la importancia del emprendimiento social como medio conductor hacia la recuperación de la dimensión altruista en el sistema económico global, en busca de un sistema más justo y eficiente.

Desde finales del siglo XX, con el proceso de globalización en marcha, se generaron muchas expectativas optimistas respecto a la evolución de la economía mundial, principalmente en aquellas instituciones que velan por el orden mundial, tales como el Fondo Monetario Internacional (FMI) o la Organización Mundial de Comercio (OMC). Se esperaba que en la globalización, al generarse mayores flujos financieros y comerciales internacionales, éstos iban a involucrar un desarrollo más rápido en las regiones atrasadas de África, Asia y América Latina. Asimismo, se esperaba un crecimiento más estable en el largo plazo y menos cíclico en aquellos países más avanzados. Los pilares de esta Nueva Economía, son las políticas de liberalización y desregulación económica iniciadas en los años 80 del siglo pasado, y la elevada competencia entre empresas, que aprovechando el uso de nuevas tecnologías de la informática y las comunicaciones, tendieron a formar grandes grupos económicos o empresas transnacionales, mediante las adquisiciones y/o fusiones. De tal modo, que para explicar el por qué el modelo que sustenta la economía global no está generando los resultados esperados en pleno siglo XXI, se ha generado un debate importante.

Así, los defensores del modelo arguyen que el problema está en las fallas del esquema político- institucional que al impedir una mayor liberalización económica limitan la creación de empresas e innovaciones, En contraste, los críticos del modelo, señalan como responsables de la gran crisis financiera internacional iniciada en 2008, al exceso de desregulación y liberalización y a la elevada competencia empresarial. (Ver Stiglitz 2012, citado en Bouby, 2013).

Es interesante la participación de Sampedro en este debate, quien plantea que la estructura de todo sistema económico tiene tres niveles, el técnico-económico referido a las reglas de funcionamiento del mercado; el socio-político referido al papel de las instituciones en la regulación del mercado; y el axiológico referido a los valores en los que se asienta el sistema de economía de mercado. Señala Sampedro que el debate para explicar las causas de la crisis global se ha orillado al nivel socio-político principalmente centrado en la controversia libertad de mercado versus intervención del mercado. Sin embargo, plantea que el debate también debe centrarse en el nivel axiológico, a fin de esclarecer que la vieja controversia entre los valores libertad e igualdad no son antagónicos, sino complementarios (Sampedro, 1983, citado en Santos, 2013).

Amartya Sen, enriquece el debate al cuestionar la separación progresiva entre ética y eco nomía. La ética, como disciplina filosófica, estudia la relación entre la acción humana y los fines que persigue, por lo que se podría plantear que una actividad humana se considera ética si persigue simultáneamente el interés individual y colectivo; es decir, cuando los valores que lo sustentan están asentados en la sociedad (Guzmán 1990, Galindo y Guzmán 2008, citados en Santos 2013). Sen argumenta que, en el caso concreto de la actividad económica, el valor que orienta el comportamiento humano es el egoísmo individual, que conduce a un afán de lucro desmedido en la competencia en el mercado, lo cual según Smith, contribuye al progreso económico guiado por una mano invisible (Sen 1987, citado en Santos 2013).

El sistema capitalista enfatiza la dimensión egoísta del comportamiento humano y prescinde de otras dimensiones como la altruista o la cooperativa, lo cual conduce a configurar un sistema económico global caracterizado en su nivel axiológico por el paradigma de la competencia; en su nivel socio-político, instituciones internacionales como el FMI y la OMC, fomentan valores como la competencia y el afán de lucro, configurando 
las reglas de oferta y demanda para el quehacer empresarial en su nivel tecno-económico, con el objetivo de maximizar beneficio coherente con la lógica de la conducta egoísta.

Este sistema de capitalismo global, caracterizado por un crecimiento cíclico de auge y crisis, viene generando grandes problemas de orden económico y social. Las sucesivas crisis del sistema, tales como las crisis energéticas de los años 70 del siglo pasado, seguida por la crisis mexicana de 1995, la crisis del sudeste asiático de 1997, la crisis rusa de 1998, la crisis argentina de 2000, la crisis japonesa que duró desde 1993 hasta 2003 y exacerbadas con la crisis financiera internacional iniciada en 2007 en Estados Unidos y extendida a partir de 2008 a todas las regiones, han dado como resultado una elevada desigualdad social y pérdida de empleo y de renta en los países desarrollados por la quiebra de empresas; y el crecimiento de la pobreza, desempleo y exclusión social en los países en desarrollo, debido a la elevación de los precios de los bienes de consumo de primera necesidad y la falta de crédito. Mostrando un sistema injusto e ineficiente al no lograr, a decir del Banco Mundial, la meta esencial de proporcionar bienestar a la población en general (Banco Mundial 2013, citado en Santos 2013).

Esta situación está contribuyendo a la cada vez mayor polarización a nivel de la economía mundial por el deterioro de las condiciones de vida de inmensas capas de la población a nivel global.

Ban Ki-moon, secretario general de la ONU, ha calificado de inaceptable el poco avance en el cumplimiento de los Objetivos de Desarrollo Mundial, entre los que se halla la reducción a la mitad de la extrema pobreza respecto al nivel que había en 1990. Califica de inaceptable que para dos tercios de la población mundial sea un lujo el acceso al agua, que además un millón de personas mueran de malaria cada año y que actualmente mil millones de personas vivan en extrema pobreza, siendo prácticamente excluidas por la economía global (Wompner 2012).

Chaparro-Africano (2010), presenta indicadores importantes de los resultados de la inadecuada y deficiente gestión económica del modelo económico imperante para atender las necesidades y problemas de la sociedad, las cuales reflejan el nivel de degradación social y ecológica actual.

Alrededor de 1,020 millones sufren de hambre según el Programa Mundial de Alimentos (PMA
2010). Más de 1,000 millones de personas en situación de pobreza extrema. Alrededor de 500 millones de pequeños agricultores que aportan la mayoría de alimentos consumidos por la población en los países en desarrollo, sufren hambre y pobreza y tienen escaso acceso a servicios sociales básicos (Dixon et al., 2001, citado en Chaparro-Africano 2010). El Índice Planeta Vivo de la biodiversidad global ha caído en casi $30 \%$ durante los últimos 35 años, (WWF 2008, citado en Chaparro-Africano 2010).

Hacia la década de los 60 del siglo XX, los países del mundo tenían la capacidad de generar la oferta suficiente para satisfacer su propia demanda. En el año 2005 muchos países solo pudieron satisfacer su demanda con la importación de otros países, debido al incremento de la población y del consumo individual. De otro lado, en la década de los 80 ya se había excedido por primera vez el uso de la biocapacidad total de la tierra, generándose deforestación, escasez de agua, disminución de la biodiversidad y cambio climático, poniéndose en riesgo el bienestar y desarrollo de los países.

\section{El emprendimiento económico como resultado de la globalización y su paradigma competitivo}

Ante un entorno económico global cíclico, cambiante que surge enarbolando los valores del paradigma de la competencia, se necesitan emprendedores que tengan capacidad de iniciativa, creatividad y sean proactivos para superar el alto riesgo que supone todo proyecto empresarial. La elevada competencia dio lugar a otras dos consecuencias influyentes en el comportamiento empresarial, la mayor incertidumbre y la fragmentación de los mercados (Carlson 1996, citado en Santos 2013)). La incertidumbre genera mayor inflación, inestabilidad de los tipos de cambio, alto desempleo y bajo crecimiento económico. La fragmentación del mercado de bienes y servicios, consecuencia de los diferentes gustos de los consumidores según sexo, edad, nivel de renta, localidad, nivel educativo, etcétera., obliga a las empresas a seguir estrategias basadas en la diferenciación. La fragmentación del mercado laboral originado por las diferencias en la calificación del trabajador y por tanto en los niveles del salario, genera en las economías en desarrollo altos niveles de informalidad (Bouby 2013).

Dichas consecuencias exigen en cualquier región de una mayor dosis de capacidad emprendedora para sobrevivir en el mercado. De esta forma, 
la globalización y su paradigma competitivo han propiciado el desarrollo de un paradigma complementario: el de la economía del emprendimiento como contraposición al paradigma de la economía de la gestión. (Audretsch y Thurik 2000, citados en Santos 2013)

El paradigma de la economía de la gestión fue el dominante hasta la crisis de 1973, bajo el modelo de estructura empresarial basado en la gran corporación con sus economías de escala, estandarización, estabilidad y control, y sometido a una política regulatoria estricta desde las administraciones públicas centrales.

Por el contrario, la economía del emprendimiento se desarrolla bajo un modelo empresarial mixto basado en la presencia de grandes corporaciones y pequeñas y medianas empresas en red, bajo un entorno de cambio, turbulencia y flexibilidad; apoyado, por una política de desregulación y estímulos, descentralizada en las administraciones públicas locales. El papel de los emprendedores en esta economía del emprendimiento es importante al contribuir al surgimiento de empresas innovadoras que crean bienes y servicios de mayor demanda, generando gran valor agregado que impulsa el proceso de crecimiento económico.

Sin embargo, el problema del sistema económico global respecto al emprendimiento, está vinculado con el tipo de emprendimiento que puede surgir. Por un lado, existen emprendimientos destructivos pues se desenvuelven en actividades especulativas y que no generan valor agregado porque solo persiguen el lucro del emprendedor en el corto plazo. Por otro lado, están los emprendimientos improductivos que generan valor agregado y maximizan beneficios en el corto plazo a través de la imitación (Santos 2013).

La presencia de dichos emprendimientos se relacionan con fallas en el sistema de incentivos institucionales, es decir, con fallas en el nivel socio-político del sistema económico (Baumol 1990, citado en Santos 2013). En el nivel axiológico del sistema, el desmedido afán de lucro es el denominador común de los emprendimientos tanto destructivo como improductivo, convirtiéndose así el emprendimiento de mercado en la correa de transmisión del valor supremo del egoísmo en el que se asienta el paradigma competitivo.

Así, existen evidencias preocupantes respecto al proceso de incubación de empresas en emprendimientos de negocios, las que subestiman las dimensiones ecológica y social al momento de establecer su viabilidad, al sólo considerar el valor agregado que éstas pueden generar (Chaparro-Africano 2009). Las raíces económicas del deterioro ecológico y social que está en la base de la actual crisis mundial, tienen una relación causal entre el fomento del emprendimiento y el empresarismo y la perpetuación del círculo vicioso de degradación ecológica y social mundial (Carpintero 1999 y Naredo 2007, citados en Chaparro-Africano 2010).

Para Wompner, emprender es lo que más movilidad social y económica crea, aún más que la educación, ya que en los últimos años ha ido disminuyendo su impacto con respecto a este propósito (Wompner 2012).

Según García, existen dos tipos de emprendimiento, el empresarial, también denominado emprendedurismo, y el emprendimiento social. Conforme a la normativa de los países latinoamericanos, no todos estos países poseen normas concretas sobre emprendimiento pero sí sobre PYME. Sin embargo, ninguna de las normas hace mención a la bioética. Aunque en abundante literatura al respecto, evidencian siete puntos de contacto entre el emprendimiento y la bioética, como son, el talento humano; la pobreza, el bien común y la solidaridad; el medio ambiente, agro y desarrollo sostenible; el manejo de los recursos económicos; el compromiso bioético con los clientes; los límites bioéticos a la innovación, y finalmente, los emprendimientos sociales.(García Arango 2014, pp. 1-4)

En suma, el problema con el emprendimiento no está en el emprendimiento per. Se, sino en la motivación que guía dicho emprendimiento.

Desde hace tiempo el emprendimiento (entrepreneurship) ha estado supeditado a la esfera empresarial, su desarrollo en otros ámbitos, como el público y el social, es incipiente. (Toca 2010)

Morris, reconoce el emprendimiento como actividad transdisciplinaria, carente de una teoría universal y soportada en teorías prestadas de la economía, la ingeniería, la historia económica y empresarial, la sociología, la administración y, recientemente, de la psicología. (Morris 1998, citado en Toca 2010). Otros autores lo identifican como campo específico de conocimiento con conceptos y metodologías aún en fase de consolidación y formación (Henry, Hill y Leith 2005; Henrique y Da Cunha 2008, citados en Toca 2010). 
Según Shane, hay tres escuelas de pensamiento que brindan teorías para realizar investigación en emprendimiento. La primera brinda teorías del equilibrio neoclásico, en las que atributos personales definen los perfiles de los emprendedores; la segunda brinda teorías psicológicas, que reconocen el emprendimiento como un proceso que depende de la habilidad y la voluntad del individuo para promover acciones; y la tercera brinda teorías austríacas, cuyo supuesto es que los individuos poseen diferente información que conduce a que visualicen distintos valores en un bien o servicio dado. (Shane 2000, citado en Toca 2010)

El emprendimiento fue limitado al contexto empresarial porque el concepto posiblemente se inspiró en la teoría económica. Contexto en el cual el desempeño de una función empresarial genera cambio económico y cualquier actividad que aumente el potencial de cambio, será por definición un acto emprendedor (Knight 1921; Schumpeter 1934 y Barzelay 2001, citados en Toca 2010). En razón a la cual probablemente Morris, desconoce la presencia del emprendimiento en organizaciones no lucrativas, en movimientos políticos e incluso en agencias del gobierno (Toca 2010).

\section{Responsabilidad social en el sector privado}

Para Milton Friedman (1970), la responsabilidad social que tienen las corporaciones es únicamente incrementar utilidades; y si ésta tiene que hacer una contribución social, estaría privando a sus accionistas de ejercer el derecho de decidir sobre sus recursos económicos (Porter y Kramer 2002, citados en Guzmán y Trujillo 2008).

La posición neo-friedmaniana argumenta que las corporaciones pueden desarrollar políticas de responsabilidad social, siempre que éstas incrementen el retorno de los accionistas; por lo cual Porter y Kramer, 2002, arguyen que cuando las corporaciones usan sus esfuerzos caritativos para mejorar su entorno competitivo empresarial, logran a su vez alcanzar beneficios sociales y económicos. (Porter y Kramer 2002, citados en Guzmán y Trujillo 2008)

Para Kolstad, a pesar de haber un acuerdo entre gerentes y propietarios de perseguir de parte de gerentes el bienestar de los propietarios, éste no exime a ninguno de ellos de propiciar la colaboración del sector privado a la solución de los problemas sociales en países en desarrollo, debido a la incapacidad de las instituciones públi- cas y de los gobiernos para hacerlo por sí solos. Para Kolstad “...la posición neo-friedmaniana no debe seguir siendo usada por no ser éticamente defendible" (Kolstad 2007, citado en Guzmán y Trujillo 2008).

Para Fassin, hay tres dimensiones principales que llevan a las personas a actuar de manera no ética. La primera, la avaricia y la persecución de utilidades; la segunda, la naturaleza de la competencia y el deseo por golpear al competidor; y la tercera, la necesidad de asegurar o restaurar un estándar de justicia (Fassin 2005, citado en Guzmán y Trujillo 2008). Pues según Rose “...los gerentes favorecen la generación de valor de los accionistas sobre las creencias éticas personales y el bien común, porque creen firmemente que las leyes corporativas exigen de ellos la toma de acciones legales para beneficiar en primera medida a los accionistas" (Rose 2007, citado en Guzmán y Trujillo 2008).

Sin embargo, la promoción y desarrollo de la responsabilidad social de parte de los gerentes en forma complementaria a su quehacer empresarial privado, buscaría el bienestar de los diferentes grupos (Chaparro-Africano, 2010).

En resumen, el modelo económico global sustenta su crecimiento en el emprendimiento económico convencional y en la institución empresarial en general, soslayando sus efectos de crecimiento sin desarrollo, generación de externalidades sociales y ecológicas que conducen cíclicamente a crisis regionales y mundiales, y que han llegado a un punto de no retorno en lo ecológico y en lo económico-social y a una gran amoralidad que significa la pobreza, desempleo, inequidad y la insustentabilidad. De hecho, son las empresas transnacionales -actores principales de la economía global- las que han agudizado dichos indicadores sociales y ecológicos, dadas sus características de empresas basadas en las relaciones capitalistas de producción; cuyo objetivo central es la maximización y acumulación de capital, cuya visión de industrialización se basa en la masiva extracción de recursos naturales y generación de deshechos. Es urgente avanzar en la conformación de emprendedores sociales e instituciones empresariales más sustentables que aporten a la multidimensionalidad del desarrollo a nivel territorial. Lo cual es concordante con un comportamiento más ético al contribuir a la solución de los problemas más acuciantes, principalmente en los países en desarrollo. 


\section{La importancia del emprendimiento social frente al proceso de globalización de la economía y de los mercados.}

El emprendimiento social surge como un fenómeno de transformación sociocultural, como medio para democratizar las actividades económicas basadas en la ayuda mutua y el esfuerzo personal. Según Souza Silva, el emprendimiento social tiene su origen en las comunidades indígenas y ha sido influenciado conforme el paso de los diversos modos de producción a través de la historia económica mundial (Souza Silva 2007, citado en García Arango 2014).

Así, en la era de la revolución industrial, se consideraba a la industria como motor del progreso, y a los factores, tales como, el humano, social, ecológico, cultural y ético, se les consideraba obstáculos al crecimiento económico, por lo tanto el emprendimiento social era percibido como innecesario. En esta época, la relación EstadoNación se materializó con el establecimiento de políticas, instituciones y roles epistemológicos concordantes con el funcionamiento del sistema capitalista y su respectivo orden social.

A partir de la década de los 70, como producto de la crisis social y económica vivida en ese lapso, se producen una serie de cambios políticos de importancia para el emprendimiento. Por ejemplo, los Estados asumen nuevas dimensiones y se pasa de una época de acción centrada en el crecimiento económico y alto emprendimiento de actores privados, a otra de preocupación social vulnerable en las acciones de políticas públicas (García y Piña Zambrano 2013).

El emprendimiento basado en el trabajo asociativo y la propiedad colectiva es de gran trascendencia en América Latina, donde los gobiernos recientemente han empezado a comprender la importancia del emprendimiento como forma de contrarrestar la pobreza y el desempleo.

La importancia del emprendimiento social, está vinculada a la consecución de un sistema económico más justo en el cual la ética y la economía van unidas (Santos 2013).

Bent-Goodiey define el emprendimiento social "como un proceso de creación de organizaciones, guiado por la ética social y basado en la integración de servicio social y habilidades en relaciones públicas" (Bent-Goodiey 2002, citado en Toca 2010). Alvord, Brown y Letts definen el emprendimiento social "como renovación para generar impacto social, perspectiva centrada en ideas y arreglos sociales con consecuencias sobre las problemáticas sociales" (Alvord, Brown y Letts 2002, citado en Toca 2010). Para Stryjan, el emprendimiento “...gira en torno a la prestación de servicios sociales y supone la obtención y manejo de recursos socialmente etiquetados, concibiendo formas para derivar servicios de ellos y garantizando una consecución sostenible en el tiempo. La actividad esencial de los emprendedores sociales se centra en movilizar contribuciones y en alinear a los contribuyentes con sociedades viables" (Stryjan 2006, citado en Toca 2010). Para Handy, Ranada y Kassam, los emprendedores sociales presentan características como voluntad a asumir riesgos, autodirección, liderazgo, innovación y conciencia del otro (Handy, Ranada y Kassam 2007, citados en Toca 2010).

La creación y desarrollo de empresas no es exclusiva de personas que persiguen el lucro. El emprendimiento social, reuniendo las características de cualquier otro emprendimiento, se distingue de los demás principalmente en que la motivación del emprendedor social es el altruismo (Leadbetter 1997; Yunus 2009 y Yunus 2011, citados en Santos 2013). "El altruismo es un valor que significa que el individuo realiza un sacrificio personal en beneficio de otros. El altruismo supone reciprocidad y es, por tanto, la base de la solidaridad entre individuos" (Santos 2013).

Adam Smith, en su libro "La teoría de los sentimientos morales" (Smith, [1756] 2004) trata sobre la teoría de la motivación del individuo en forma más compleja de la que se expone en la teoría económica ortodoxa. Smith, señala "en primer lugar que, es el amor propio (self-love) o consideración y estima hacia uno mismo (autoestima), lo que mueve la acción humana. En segundo lugar, afirma que dicho amor propio tiene dos dimensiones, la egoísta (el interés propio) y la altruista, siendo esta última consecuencia de lo que se denomina "simpatía", es decir, la comprensión afectiva entre individuos" (Santos 2013).

El emprendimiento social se basa en el altruismo para generar valor social con el fin de atender necesidades sociales tales como la pobreza, la exclusión social, el desempleo de personas con discapacidades físicas o psíquicas o el deterioro medioambiental. Dichas necesidades se atienden produciendo bienes y servicios de forma inno- 
vadora (Drayton 2002, citado en Santos 2013) $\mathrm{y}$, no necesariamente, mediante la creación y desarrollo de empresas (Boschee, 1995; Defourny y Nyssen, 2012, citados en Santos 2013). En tal sentido, como en cualquier actividad económica es necesaria también la obtención de un beneficio secundario, pero no con el objetivo de repartir dividendos sino de viabilizar la reinversión que permita la mejora continua de la actividad y una autosuficiencia financiera que no la haga depender de ayudas públicas o dinero filantrópico (Yunus 2011, citado en Santos 2013).

Existen dos tipos de emprendimientos: los emprendimientos económicos o de mercado y los emprendimientos sociales, y entre estas dos posiciones pueden haber algunos tipos híbridos. Un ejemplo de un emprendimiento social híbrido son las cooperativas, en las que existe un objetivo de lucro pero que a su vez tienen un contenido social en el desarrollo de su actividad. Ejemplo de emprendimiento de mercado híbrido es una organización capitalista de sociedad anónima que, buscando el máximo beneficio en su actividad, establece un programa de Responsabilidad Social Empresarial (RSE), pudiéndose denominarse emprendimiento de mercado con compromiso social.

El emprendimiento social puro es aquel que antepone su misión social al objetivo del beneficio. En función de su autosuficiencia financiera existen dos tipos de emprendimiento social: emprendimiento social de no mercado cuando no cubre sus costos y depende de ayudas públicas o filantrópicas, ejemplo las ONG; y emprendimiento social de mercado cuando generan ingresos y cubren sus costos y pueden o no tener afán de lucro, ejemplo el emprendimiento individual del Grameen Bank (Yunus 2012, citado en Santos 2013).

Uno de los activos más importantes del emprendimiento social, aparte de su objetivo social, es el capital social. Según Bourdieu, “...el capital social es la suma de todos los recursos potenciales y reales que se acumulan en un individuo o grupo de individuos debido a la posesión de una red duradera de relaciones más o menos institucionalizadas de conocimiento y reconocimiento mutuo" (Bourdieu 1986, citado en Santos 2013). Como consecuencia de que el altruismo y la reciprocidad son bases del emprendimiento social, es lógico que el capital social sea su activo principal. El capital social “....tanto en su dimensión cognitiva (confianza) como en su dimensión estructural (redes de contacto), permite a todo emprendimiento acceder a otras formas de capital, ya sea físico, humano o financiero. De esta forma, los emprendimientos sociales suplen las carencias financieras que tienen para iniciar la actividad". (Leadbetter 1997; Linan y Santos 2007, citados en Santos 2013).

El emprendimiento social está surgiendo como una respuesta a los problemas que está generando el modelo de capitalismo global a la población, fundamentalmente en términos de desigualdad, pobreza y problemas medio ambientales; y para cubrir el vacío de las débiles políticas sociales de los gobiernos enfocados en políticas de desregulación y liberalización económica.

Bajo este entorno surgen grandes necesidades sociales, tales como la creación de empleo en áreas sociales deprimidas, la mayor integración laboral de las mujeres jefas de familia, la atención sanitaria a discapacitados y otras personas dependientes, la calificación de excluidos sociales, la atención a problemas de financiación de los más pobres, la atencion de problemas medioambientales, etcétera (Perrini 2006; Thompson 2002; Thompson y Doherty 2006; Yunus 2012, citados en Santos 2013).

El emprendimiento social es un modelo empresarial complementario al emprendimiento económico o de mercado, al actuar en áreas donde no suele actuar el emprendimiento de mercado, por lo cual puede contribuir a mejorar el sistema económico global en un sistema más justo y eficiente (Yunus 2009, citado en Santos 2013).

\section{CONCLUSIONES}

1. La economía global se ha ido transformando durante las últimas décadas, configurándose actualmente un nuevo modelo, basado fundamentalmente en la competencia y la nueva tecnología. Este modelo no ha logrado resolver los problemas generados en la economía global; por el contrario, los ha agudizado. Para explicar el por qué el modelo que sustenta la economía global no está generando los resultados esperados en pleno siglo XXI, se ha generado un debate importante.

2. Los defensores del modelo arguyen que el problema está en las fallas del esquema político-institucional que, al impedir una mayor liberalización económica limitan la creación 
de empresas e innovaciones. En contraste, los críticos del modelo señalan como responsables de la gran crisis financiera internacional iniciada en 2008, al exceso de desregulación y liberalización y a la elevada competencia empresarial.

3. El sistema capitalista enfatiza la dimensión egoísta del comportamiento humano y prescinde de otras dimensiones como la altruista o la cooperativa, lo cual conduce a configurar un sistema económico global caracterizado en su nivel axiológico por el paradigma de la competencia; en su nivel socio-político, instituciones internacionales como el FMI y la $\mathrm{OMC}$, fomentan valores como la competencia y el afán de lucro, configurando las reglas de oferta y demanda para el quehacer empresarial en su nivel tecno-económico, con el objetivo de maximizar beneficio coherente con la lógica de la conducta egoísta.

4. Esta situación está contribuyendo a la cada vez mayor polarización a nivel de la economía mundial por el deterioro de las condiciones de vida de inmensas capas de la población a nivel global.

5. Ante este entorno económico global cíclico, cambiante, que surge enarbolando los valores del paradigma de la competencia, se necesita emprendedores que tengan capacidad de iniciativa, creatividad y sean proactivos para superar el alto riesgo que supone todo proyecto empresarial.

6. Sin embargo, el problema del sistema económico global respecto al emprendimiento está vinculado con el tipo de emprendimiento que puede surgir. La importancia del emprendimiento social está vinculada a la consecución de un sistema económico más justo en el cual la ética y la economía van unidas.

7. El emprendimiento social es un modelo empresarial complementario al emprendimiento económico o de mercado al actuar en áreas donde no suele actuar el emprendimiento de mercado, por lo cual puede contribuir a mejorar el sistema económico global en un sistema más justo y eficiente.

\section{LITERATURA CITADA}

Bouby T, Francisca. (2013). "Gestión estratégica e innovación en las pymes peruanas bajo el actual contexto de crisis económica internacional". En: Gestión en el Tercer Milenio, Revista de Investigación de la Facultad de Ciencias Administrativas de la UNMSM. Vol. 16, № 32. UNMSM, Lima.

Castellanos D., Oscar Fernando; Chávez P., Renzo David; y Jiménez H., Claudia Nelcy. (2003). "Propuesta de formación en liderazgo y emprendimiento". En: Innovar, 13 (22), 145-156. Fecha de consulta: 04/11/2014. Disponible en: <http://www.scielo.org.co/ scielo.php?script $=$ sci_arttext\&pid $=$ S0121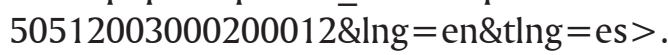

Chaparro-Africano, Adriana. (2010). "Emprendimientos y empresas agrarias y rurales más sustentables, para la sustentabilidad de los territorios". En: Agronomía Colombiana, 28 (3), 471-478. Fecha de consulta: 04/11/2014. Disponible en: <http://www.scielo.org.co/ scielo.php?script $=$ sci_arttext\&pid $=$ S0120$99652010000300015 \& \operatorname{lng}=$ en\&tlng $=e s>$.

García Arango, Gustavo Adolfo. (2014). "Bioética, emprendimiento e innovación en el contexto latinoamericano". En: Rev. Lasallista Investig. [serial on the Internet]. $2014 \mathrm{Jan}, 11$ ( 1 ): 110-118. Fecha de consulta: 04/11/2014 . Disponible en: <http://www.scielo.org.co/ scielo.php?script $=$ sci_arttext\&pid $=$ S1794$44492014000100014 \& \operatorname{lng}=\mathrm{en}>$.

García, Bismania; Piña-Zambrano, Henri. (2013). "Institucionalidad venezolana sobre emprendimiento social durante el periodo 1999-2010". En: Agricultura, Sociedad y Desarrollo, 10 (2), 215-234. Fecha de consulta: 04/11/2014. Disponible en: <http://www.scielo.org.mx/ scielo.php?script $=$ sci_arttext\&pid $=$ S1870$54722013000200005 \& \operatorname{lng}=\mathrm{es} \& \operatorname{tlng}=\mathrm{es}>$.

Guzmán Vásquez, Alexander; y Trujillo Dávila, María Andrea. (2008). "Emprendimiento Social - Revisión de Literatura". En: Estudios Gerenciales, 24(109), 105-123. Fecha de consulta: 04/11/2014. Disponible en: <http://www.scielo.org.co/scielo. php? script $=$ sci_arttext $\&$ pid $=$ S0123$59232008000400005 \& \operatorname{lng}=$ en\&tlng $=\mathrm{es}>$.

Santos Francisco, J.; Barroso, María de la O.; Guzmán, Carmen. (2006). "La Economía global y los emprendimientos sociales". Revista de Economía Mundial, núm. 35, pp. 177-196, 
Sociedad de Economía Mundial, Huelva, España. Disponible en: http://www.redalyc. org/articulo.oa?id $=86629567010$

Toca Torres, Claudia. (2010). "Consideraciones para la formación en emprendimiento: Explorando nuevos ámbitos y posibilidades". En: Estudios Gerenciales, 26 (117), 41-60. Fecha de consulta: 04/11/2014. Disponible en: < http://www.scielo.org.co/ scielo.php?script $=$ sci_arttext\&pid $=$ S0123$59232010000400003 \& \operatorname{lng}=$ en\&tlng $=e s>$.

Wompner Gallardo, F. (2013). El emprendimiento como factor de movilización social. Nómadas. Revista Crítica de Ciencias Sociales y Jurídicas, 36(4), 375-380. Disponible en: http://revistas.ucm.es/index.php/NOMA/ article/view/42361. 